\title{
Correction: Effect of high milk and sugar-sweetened and noncaloric soft drink intake on insulin sensitivity after 6 months in overweight and obese adults: a randomized controlled trial
}

\author{
Sara Engel · Tine Tholstrup · Jens M. Bruun · Arne Astrup · Bjørn Richelsen · Anne Raben
}

Published online: 22 November 2019

(c) Springer Nature Limited 2019

Correction to: European Journal of Clinical Nutrition (2018) 72:358-366

https://doi.org/10.1038/s41430-017-0006-9
Since publication the authors noticed an error in Tables 2, 3, and 4 of the original article, where the preintervention values were presented by mean and SD instead of mean and $\mathrm{SE}$ as described in the table text. The correct tables are reproduced below where SD's are replaced by SE's.

The original article can be found online at https://doi.org/10.1038/ s41430-017-0006-9. 
Table 2 Sex, age and anthropometric measurements and body composition at baseline, after 6 months intervention and differences between the beverage groups ${ }^{\mathrm{a}}$

\begin{tabular}{llllll}
\hline & Milk & NCSD & SSSD & Water & $P$ \\
\hline Sex $[n(\%)]$ & $15(25)$ & $15(25)$ & $14(23.3)$ & $16(26.7)$ & \\
Women & $11(18.3)$ & $12(20)$ & $6(10)$ & $11(18.3)$ & \\
Men & $4(6.7)$ & $3(5)$ & $8(13.3)$ & $5(8.3)$ & \\
${ }^{b}$ Age, years & $37.7 \pm 9.1$ & $39 \pm 7.6$ & $37.8 \pm 8.0$ & $39 \pm 7.3$ & 0.94 \\
Body weight, kg & & & & & \\
$\quad$ Pre intervention & $94.0 \pm 4.4$ & $94.5 \pm 3.3$ & $94.9 \pm 3.1$ & $98.4 \pm 5.4$ & 0.87 \\
$\quad$ Post intervention & $95.5 \pm 4.3$ & $95.0 \pm 3.3$ & $96.4 \pm 3.0$ & $99.1 \pm 5.8$ & 0.43 \\
BMI, kg/m ${ }^{2}$ & & & & & \\
$\quad$ Pre intervention & $31.4 \pm 0.8$ & $33.4 \pm 1.1$ & $30.8 \pm 0.7$ & $31.5 \pm 1.1$ & 0.25 \\
Post intervention & $32.0 \pm 0.8$ & $33.6 \pm 1.1$ & $31.3 \pm 0.7$ & $31.7 \pm 1.1$ & 0.30
\end{tabular}

Fat mass, kg

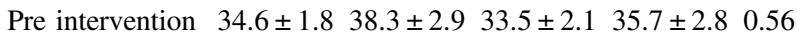

$\begin{array}{lllll}\text { Post intervention } 35.9 \pm 2.4 & 38.5 \pm 2.8 & 35.6 \pm 2.3 & 35.9 \pm 3.3 & 0.27\end{array}$

Fat free mass, $\mathrm{kg}$

$\begin{array}{lllll}\text { Pre intervention } 56.7 \pm 3.6 & 53.6 \pm 2.7 & 59.7 \pm 3.1 & 56.3 \pm 3.2 & 0.64\end{array}$

$\begin{array}{lllll}\text { Post intervention } 57.3 \pm 3.8 & 54.1 \pm 2.8 & 58.4 \pm 3.1 & 56.4 \pm 3.3 & 0.34\end{array}$

The subjects were randomly assigned to the four groups of 1 daily $\mathrm{L}$ of test beverage

NCSD noncaloric soft drink, SSSD sugar-sweetened soft drink

${ }^{a}$ All values are means \pm SEs. Statistical differences were analysed in an ANCOVA model with gender and values from baseline included as covariates. $n=60$ ( $n=58$ for fat mass and fat free mass due to two subjects had missing values)

${ }^{\mathrm{b}}$ For age values are means \pm SDs and difference analyzed by ANOVA 
Table 3 OGTT values, fasting values, and blood pressure at baseline, after 6 months intervention and differences between the beverage groups ${ }^{\mathrm{a}}$

\begin{tabular}{|c|c|c|c|c|c|}
\hline & Milk & NCSD & SSSD & Water & $P$ \\
\hline \multicolumn{6}{|c|}{ AUC OGTT glucose, mmol/L } \\
\hline Pre intervention & $894 \pm 75$ & $864 \pm 55$ & $889 \pm 61$ & $829 \pm 51$ & 0.86 \\
\hline Post intervention & $911 \pm 82$ & $876 \pm 61$ & $883 \pm 60$ & $812 \pm 62$ & 0.86 \\
\hline \multicolumn{6}{|c|}{ AUC OGTT insulin, pmol/L } \\
\hline Pre intervention & $36745 \pm 8756$ & $32396 \pm 3177$ & $24834 \pm 2354$ & $25784 \pm 2772$ & 0.30 \\
\hline Post intervention & $27945 \pm 6662$ & $22766 \pm 3090$ & $24364 \pm 4889$ & $17786 \pm 2726$ & 0.49 \\
\hline \multicolumn{6}{|c|}{ Fasting glucose, $\mathrm{mmol} / \mathrm{L}$} \\
\hline Pre intervention & $5.42 \pm 0.18$ & $5.52 \pm 0.12$ & $5.48 \pm 0.14$ & $5.26 \pm 0.13$ & 0.59 \\
\hline Post intervention & $5.69 \pm 0.27$ & $5.49 \pm 0.15$ & $5.62 \pm 0.18$ & $5.35 \pm 0.15$ & 0.47 \\
\hline \multicolumn{6}{|c|}{ Fasting insulin, $\mathrm{pmol} / \mathrm{L}$} \\
\hline Pre intervention & $81.68 \pm 18.37$ & $75.35 \pm 7.91$ & $55.32 \pm 6.12$ & $68.09 \pm 13.67$ & 0.52 \\
\hline Post intervention & $91.84 \pm 19.40$ & $74.73 \pm 10.13$ & $61.10 \pm 6.77$ & $93.19 \pm 24.94$ & 0.73 \\
\hline \multicolumn{6}{|l|}{ Matsuda index } \\
\hline Pre intervention & $8.85 \pm 1.67$ & $6.01 \pm 0.92$ & $8.08 \pm 1.22$ & $7.98 \pm 1.05$ & 0.42 \\
\hline Post intervention & $7.88 \pm 1.59$ & $6.14 \pm 0.84$ & $6.58 \pm 0.74$ & $8.38 \pm 1.27$ & 0.79 \\
\hline \multicolumn{6}{|l|}{ HOMA-IR ${ }^{b}$} \\
\hline Pre intervention & $1.55 \pm 0.35$ & $1.44 \pm 0.15$ & $1.06 \pm 0.12$ & $1.29 \pm 0.26$ & 0.53 \\
\hline Post intervention & $1.76 \pm 0.38$ & $1.42 \pm 0.19$ & $1.17 \pm 0.13$ & $1.71 \pm 0.44$ & 0.56 \\
\hline \multicolumn{6}{|l|}{ HOMA-IR AUC } \\
\hline Pre intervention & $2.95 \pm 0.76$ & $2.58 \pm 0.31$ & $2.12 \pm 0.22$ & $3.08 \pm 0.54$ & 0.53 \\
\hline Post intervention & $3.60 \pm 0.98$ & $2.58 \pm 0.39$ & $2.12 \pm 0.25$ & $3.08 \pm 0.82$ & 0.65 \\
\hline \multicolumn{6}{|l|}{ PAI-1, ng/mL } \\
\hline Pre intervention & $55.91 \pm 9.6$ & $75.90 \pm 16.6$ & $50.48 \pm 9.3$ & $44.62 \pm 10.9$ & 0.29 \\
\hline Post intervention & $46.1 \pm 7.8$ & $49.4 \pm 9.0$ & $52.9 \pm 13.3$ & $41.2 \pm 6.6$ & 0.60 \\
\hline \multicolumn{6}{|l|}{ FFA, mmol/L } \\
\hline Pre intervention & $0.59 \pm 0.05$ & $0.63 \pm 0.04$ & $0.53 \pm 0.06$ & $0.60 \pm 0.05$ & 0.58 \\
\hline Post intervention & $0.50 \pm 0.05$ & $0.62 \pm 0.07$ & $0.55 \pm 0.05$ & $0.54 \pm 0.06$ & 0.33 \\
\hline \multicolumn{6}{|l|}{ Cholesterol, $\mathrm{mmol} / \mathrm{L}$} \\
\hline \multicolumn{6}{|l|}{ Total } \\
\hline Pre intervention & $5.04 \pm 0.28$ & $5.45 \pm 0.22$ & $4.84 \pm 0.22$ & $5.28 \pm 0.19$ & 0.28 \\
\hline Post intervention & $5.17 \pm 0.23^{\mathrm{a}, \mathrm{b}}$ & $4.95 \pm 0.18^{\mathrm{a}}$ & $5.25 \pm 0.28^{b}$ & $5.30 \pm 0.19^{\mathrm{a}, \mathrm{b}}$ & 0.01 \\
\hline \multicolumn{6}{|l|}{$\mathrm{LDL}$} \\
\hline Pre intervention & $3.17 \pm 0.25$ & $3.50 \pm 0.25$ & $3.07 \pm 0.22$ & $3.38 \pm 0.17$ & 0.53 \\
\hline Post intervention & $3.21 \pm 0.20$ & $3.17 \pm 0.18$ & $3.32 \pm 0.23$ & $3.48 \pm 0.17$ & 0.10 \\
\hline \multicolumn{6}{|l|}{ HDL } \\
\hline Pre intervention & $1.16 \pm 0.07$ & $1.18 \pm 0.08$ & $1.16 \pm 0.06$ & $1.15 \pm 0.07$ & 0.99 \\
\hline Post intervention & $1.23 \pm 0.08$ & $1.17 \pm 0.07$ & $1.21 \pm 0.09$ & $1.21 \pm 0.07$ & 0.10 \\
\hline \multicolumn{6}{|l|}{ Total:HDL } \\
\hline Pre intervention & $4.54 \pm 0.35$ & $4.83 \pm 0.30$ & $4.26 \pm 0.21$ & $5.09 \pm 0.64$ & 0.55 \\
\hline Post intervention & $4.41 \pm 0.30$ & $4.41 \pm 0.28$ & $4.49 \pm 0.24$ & $4.64 \pm 0.40$ & 0.49 \\
\hline \multicolumn{6}{|c|}{ Triacylglycerol, mmol/L } \\
\hline Pre intervention & $1.58 \pm 0.24$ & $1.73 \pm 0.16$ & $1.35 \pm 0.20$ & $1.67 \pm 0.19$ & 0.57 \\
\hline Post intervention & $1.61 \pm 0.21^{\mathrm{a}, \mathrm{b}}$ & $1.37 \pm 0.11^{\mathrm{b}}$ & $1.60 \pm 0.23^{\mathrm{a}}$ & $1.36 \pm 0.13^{\mathrm{b}}$ & 0.02 \\
\hline \multicolumn{6}{|l|}{ Blood pressure, $\mathrm{mmHg}$} \\
\hline \multicolumn{6}{|l|}{ Systolic } \\
\hline Pre intervention & $124.6 \pm 4.0$ & $131.5 \pm 3.8$ & $123.4 \pm 2.4$ & $124.2 \pm 2.7$ & 0.29 \\
\hline Post intervention & $121.2 \pm 3.1$ & $126.8 \pm 2.4$ & $125.9 \pm 2.9$ & $124.1 \pm 2.4$ & 0.18 \\
\hline
\end{tabular}


Table 3 (continued)

\begin{tabular}{lllrr}
\hline & Milk & NCSD & SSSD & Water \\
\hline Diastolic & & & & \\
$\quad$ Pre intervention & $76.0 \pm 2.3$ & $81.2 \pm 2.1$ & $74.4 \pm 2.6$ & $74.9 \pm 2.2$ \\
Post intervention & $72.8 \pm 2.4$ & $77.4 \pm 2.3$ & $77.5 \pm 2.0$ & $75.5 \pm 2.2$ \\
\hline
\end{tabular}

Statistical differences were analyzed in an ANCOVA model with Tukey pairwise comparisons adjusted for pre intervention and the covariates; age, gender, baseline BMI, and change in FM $(\mathrm{kg})$. Values that have no superscript in common are significantly different from each other (Tukey's HSD, $P<0.05)$

NCSD noncaloric soft drink, SSSD sugar-sweetened soft drink

${ }^{a}$ All values are unadjusted means \pm SEs, $n=58$ because two subjects had missing values for change in FM

${ }^{\mathrm{b}} n=57$ because one observation was considered an outlier NCSD

Table 4 Average daily consumption of energy and macronutrients at baseline and at the end of the 6 months intervention and difference between the four beverage groups $^{\mathrm{a}}$

\begin{tabular}{|c|c|c|c|c|c|}
\hline & Milk & NCSD & SSSD & Water & $P$ \\
\hline \multicolumn{6}{|l|}{ Total energy, kJ } \\
\hline Pre intervention & $9931 \pm 681$ & $9592 \pm 674$ & $10834 \pm 735$ & $10424 \pm 591$ & 0.58 \\
\hline Post intervention & $10830 \pm 889$ & $9495 \pm 660$ & $9657 \pm 890$ & $10638 \pm 806$ & 0.14 \\
\hline \multicolumn{6}{|l|}{ Fat, \% of energy } \\
\hline Pre intervention & $33.1 \pm 1.6$ & $32.4 \pm 0.9$ & $32.7 \pm 1.0$ & $34.6 \pm 1.2$ & 0.56 \\
\hline Post intervention & $32.8 \pm 1.2^{\mathrm{b}}$ & $34.2 \pm 1.4^{\mathrm{a}, \mathrm{b}}$ & $31.3 \pm 1.0^{\mathrm{b}}$ & $38.5 \pm 1.0^{\mathrm{a}}$ & $<0.01$ \\
\hline \multicolumn{6}{|l|}{ Total fat, $\mathrm{g}$} \\
\hline Pre intervention & $89.4 \pm 8.0$ & $84.4 \pm 6.9$ & $95.0 \pm 6.8$ & $99.0 \pm 8.2$ & 0.53 \\
\hline Post intervention & $96.7 \pm 10.0$ & $87.6 \pm 6.8$ & $81.3 \pm 7.5$ & $112.5 \pm 11.3$ & 0.09 \\
\hline \multicolumn{6}{|l|}{ Saturated fat } \\
\hline Pre intervention & $32.1 \pm 3.3$ & $27.5 \pm 2.6$ & $31.1 \pm 2.7$ & $32.8 \pm 3.5$ & 0.61 \\
\hline Post intervention & $37.8 \pm 4.1$ & $28.7 \pm 2.4$ & $24.9 \pm 2.2$ & $36.7 \pm 4.3$ & 0.05 \\
\hline \multicolumn{6}{|l|}{ Monounsaturated fat } \\
\hline Pre intervention & $26.8 \pm 2.4$ & $24.8 \pm 2.8$ & $26.7 \pm 2.2$ & $27.7 \pm 2.6$ & 0.87 \\
\hline Post intervention & $27.1 \pm 2.8$ & $24.8 \pm 2.0$ & $22.0 \pm 1.9$ & $32.9 \pm 3.9$ & 0.06 \\
\hline \multicolumn{6}{|l|}{ Polyunsaturated fat } \\
\hline Pre intervention & $10.8 \pm 0.8$ & $12.2 \pm 1.2$ & $12.4 \pm 0.8$ & $13.3 \pm 0.9$ & 0.36 \\
\hline Post intervention & $12.2 \pm 1.2$ & $13.4 \pm 1.1$ & $11.4 \pm 1.0$ & $16.8 \pm 1.8$ & 0.13 \\
\hline \multicolumn{6}{|c|}{ Carbohydrate, $\%$ of energy } \\
\hline Pre intervention & $48.2 \pm 1.6$ & $48.2 \pm 1.4$ & $52.1 \pm 1.6$ & $48.5 \pm 1.3$ & 0.20 \\
\hline Post intervention & $47.0 \pm 1.0^{\mathrm{a}}$ & $46.4 \pm 1.1^{\mathrm{a}}$ & $53.8 \pm 1.9^{\mathrm{b}}$ & $44.0 \pm 1.3^{\mathrm{a}}$ & $<0.001$ \\
\hline \multicolumn{6}{|l|}{ Protein, $\%$ of energy } \\
\hline Pre intervention & $16.7 \pm 5.2$ & $16.2 \pm 5.8$ & $14.1 \pm 7.2$ & $14.8 \pm 4.1$ & 0.06 \\
\hline Post intervention & $17.9 \pm 0.6^{\mathrm{a}}$ & $15.3 \pm 0.6^{\mathrm{b}, \mathrm{c}}$ & $12.9 \pm 0.8^{\mathrm{b}}$ & $15.6 \pm 0.6^{\mathrm{a}, \mathrm{c}}$ & $<0.001$ \\
\hline \multicolumn{6}{|l|}{ Calcium, mg } \\
\hline Pre intervention & $1355 \pm 116^{\mathrm{b}}$ & $983 \pm 994^{\mathrm{a}}$ & $958 \pm 89^{\mathrm{a}}$ & $950 \pm 75^{\mathrm{a}}$ & 0.01 \\
\hline Post intervention & $1848 \pm 112^{\mathrm{b}}$ & $1021 \pm 128^{\mathrm{a}}$ & $671 \pm 91^{\mathrm{a}}$ & $845 \pm 62^{\mathrm{a}}$ & 0.000 \\
\hline \multicolumn{6}{|l|}{ Alcohol, g } \\
\hline Pre intervention & $6.9 \pm 2.2$ & $11.6 \pm 4.1$ & $4.3 \pm 1.8$ & $8.3 \pm 2.6$ & 0.37 \\
\hline Post intervention & $9.7 \pm 2.8$ & $12.4 \pm 2.9$ & $7.7 \pm 4.0$ & $7.7 \pm 2.2$ & 0.82 \\
\hline
\end{tabular}

All values are means \pm SEs. Statistical differences were analysed in an ANCOVA model with baseline diet registration from before intervention included as covariates. Values that have no superscript in common are significantly different from each other (Tukey's HSD, $P<0.05$ ). $n=53$ (two subjects had no dietary records done and five had nonsufficient records). Data were assessed with a 7-day weighted dietary record estimated by using Dankost Pro dietary assessment software (Dankost)

$N C S D$ noncaloric soft drink, SSSD sugar-sweetened soft drink 